\title{
OPEN Effects of exercise on kidney and physical function in patients with non-dialysis chronic kidney disease: a systematic review and meta-analysis
}

\begin{abstract}
Keisuke Nakamura ${ }^{1,6 \bowtie}$, Tomohiro Sasaki ${ }^{1}$, Shuhei Yamamoto ${ }^{2}$, Hiroto Hayashi ${ }^{3}$, Shinji Ako ${ }^{4}$ \& Yuu Tanaka ${ }^{5}$

Patients with non-dialysis chronic kidney disease (CKD) are at greater risk of early mortality and decreased physical function with an advance in the stage of CKD. However, the effect of exercise in these patients is unclear. This meta-analysis aimed to determine the effects of physical exercise training on the risk of mortality, kidney and physical functions, and adverse events in patients with non-dialysis CKD. The meta-analysis conformed to the Preferred Reporting Items for Systematic Reviews and Meta-Analysis (PRISMA) statement and the Cochrane Handbook recommendations. On 16 August 2019, the PubMed, CINAHL, Cochrane Library databases, and Embase were electronically searched, with no restrictions for date/time, language, document type, or publication status, for eligible randomized controlled trials (RCTs) investigating the effects of exercise on mortality and kidney and physical function in patients with non-dialysis CKD. Eighteen trials (28 records), including 848 patients, were analyzed. The effects of exercise on all-cause mortality and estimated glomerular filtration rate were not significantly different from that of usual care. Exercise training improved peak/ maximum oxygen consumption compared to usual care. Regular exercise improves physical and walking capacity for patients with non-dialysis CKD. Effect on leg muscle strength was unclear.
\end{abstract}

Chronic kidney disease (CKD) is a major clinical condition affecting a significant number of individuals worldwide; additionally, it is associated with high-risk cardiovascular disease (CVD), stroke, frailty, and mortality ${ }^{1-6}$. Furthermore, there is a significant association between the severity of CKD and health care costs ${ }^{7}$. For these reasons, optimal management of CKD is especially important to prevent kidney failure, extend healthy life expectancy, and have a positive impact on health care costs.

The primary strategies for the prevention and treatment of CKD include lifestyle changes and pharmacological approaches, including the promotion of exercise, dietary changes, and antihypertensive drugs ${ }^{2}$. Studies have shown that physical function and performance in pre-dialysis CKD decreased with an advance in the stage of CKD, which may be caused by several factors, including decreased kidney function, chronic inflammation, and arteriosclerosis $^{8-10}$. A systematic review reported that improved physical function and greater levels of physical activity in pre-dialysis CKD reduced all-cause and cardiovascular mortality risk ${ }^{11}$; this highlights the important role of exercise interventions for improvement of physical function and activity levels in this population. Several systematic reviews have reported that exercise training, including both aerobic and resistance exercises, has significant positive effects on physical fitness, including physical and functional capacity, muscle strength, and blood pressure in patients with $\mathrm{CKD}^{12-14}$. However, these reviews included participants receiving dialysis therapy. There has been no systematic review or meta-analysis evaluating the effects of aerobic and resistance exercise on kidney and physical functions, adverse events, and mortality in patients with non-dialysis CKD. A

${ }^{1}$ Department of Rehabilitation, Matsumoto City Hospital, Nagano, Japan. ${ }^{2}$ Department of Rehabilitation, Shinshu University Hospital, Nagano, Japan. ${ }^{3}$ Department of Public Health, Graduate School of Medical and Dental Sciences, Tokyo Medical and Dental University, Tokyo, Japan. ${ }^{4}$ Department of Internal Medicine, Kidney \& dialysis center, Matsumoto City Hospital, Nagano, Japan. ${ }^{5}$ Department of Anesthesiology, Nara Medical University, Nara, Japan. ${ }^{6}$ Present address: Department of Rehabilitation, Matsumoto City Hospital, 4417-180 Hata, Matsumoto, Nagano 390-1401, Japan. ${ }^{\circledR}$ email: keipons55@yahoo.co.jp 


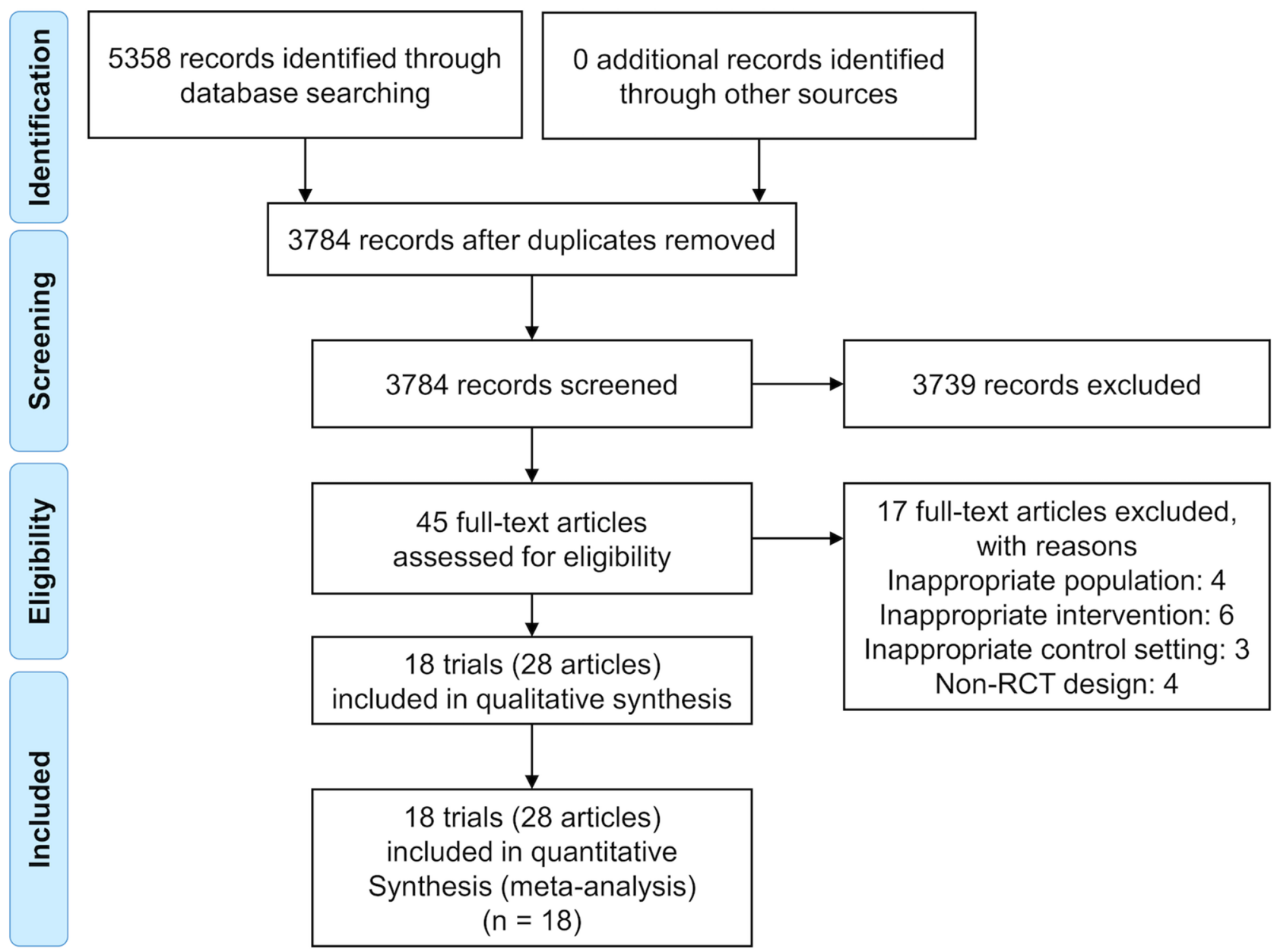

Figure 1. Study flow diagram.

systematic review conducted in 2014 reported differences in the effect of exercise training on aerobic capacity, muscular functioning, and health-related quality of life that may depend on CKD stage, dialysis treatment, and history of a kidney transplant. However, that systematic review featured only one clinical trial including patients with CKD stages $2-5$, and it was impossible to conduct a meta-analysis ${ }^{13}$. Since 2014 , there have been various RCTs evaluating the effects of physical exercise on patients with non-dialysis $\mathrm{CKD}^{15,16}$. The investigation of the effects of exercise on physical function in patients with non-dialysis CKD is clinically important because there are differences between dialysis and non-dialysis patients, including glomerular filtration rate (GFR), risk of mortality and lifestyle habits ${ }^{2,3}$.

Therefore, this systematic review and meta-analysis aimed to determine the effects of physical exercise training on risk of mortality, kidney function, adverse events, and physical function outcomes [i.e., exercise tolerance (peak/maximum oxygen consumption $\left[\mathrm{VO}_{2}\right]$ ), walking ability (6-min walk distance), and lower extremity muscle strength] in adult patients with non-dialysis CKD.

\section{Results}

In total, 3784 records were identified after the removal of duplicates, and 45 records remained after the screening of titles and abstracts. Further, 17 records were excluded based on the full eligibility criteria. In total, 18 trials (28 records $)^{8,15,17-42}$ including 848 patients with non-dialysis CKD who had met the eligibility criteria of this review were included in the analysis (Fig. 1). The characteristics of the trials included in this review are described in Table 1. Overall, the records demonstrated a broad range of follow-up duration (median follow-up $=20.5$ weeks; range 8-72 weeks) and CKD stage of the trial population (CKD stages 3-4, 9 trials; stages 2-4, 4 trials; stage 3, 1 trial; stages 3-5, 1 trial; stages 1-3,1 trial; not reported, 2 trials). Categorizations of the types of exercise training in the trials were as follows: center-based exercise $=9$ trials, home-based exercise $=4$ trials, combined both center and home-based exercise $=6$ trials, aerobic exercise $=8$ trials, resistance exercise $=2$ trials, and combined both aerobic and resistance training $=8$ trials.

Quality assessment. The results of the risk of bias assessment in all the trials are summarized in Table 2. All participants were classified into the exercise training and the usual care groups. Blinding of participants was not possible due to the nature of exercise training. Three trials $s^{17,32,36}$ showed high risks of bias related to the 


\begin{tabular}{|c|c|c|c|c|c|c|c|c|c|c|c|}
\hline \multirow[b]{2}{*}{ Trials } & \multirow[b]{2}{*}{$\begin{array}{l}N \text { (analyzed } \\
\text { Exp/Con) }\end{array}$} & \multicolumn{5}{|l|}{ Participants } & \multirow[b]{2}{*}{$\begin{array}{l}\text { Control } \\
\text { group }\end{array}$} & \multirow[b]{2}{*}{$\begin{array}{l}\text { Intervention } \\
\text { group }\end{array}$} & \multirow[b]{2}{*}{$\begin{array}{l}\text { Compliance of } \\
\text { intervention } \\
\text { group (\%) }\end{array}$} & \multirow[b]{2}{*}{$\begin{array}{l}\text { Outcome } \\
\text { measures }\end{array}$} & \multirow[b]{2}{*}{$\begin{array}{l}\text { Follow-up } \\
\text { assessment }\end{array}$} \\
\hline & & Age (years) & BMI & CKD stage & $\begin{array}{l}\text { eGFR }(\mathrm{mL} / \\
\mathrm{min} / 1.73 \\
\left.\mathrm{~m}^{2}\right)\end{array}$ & $\begin{array}{l}\text { Percentage } \\
\text { of DM (\%) }\end{array}$ & & & & & \\
\hline $\begin{array}{l}\text { Kirkman }{ }^{17} 2019 \text {, } \\
\text { USA }\end{array}$ & $16 / 15$ & 58 & 32 & Stage 3-5 & 44 & ND & Routine care & \begin{tabular}{|l|} 
Centre-based \\
aerobic \\
exercise for \\
12 weeks \\
Type: cycling, \\
walking, jog- \\
ging, ellipti- \\
cal machine \\
Frequency: 3 \\
times/week \\
Intensity: \\
$60-85 \%$ \\
HRR, RPE \\
$12-16$ \\
Duration: \\
45 min \\
\end{tabular} & 92 & $\begin{array}{l}\mathrm{VO}_{2} \text { peak, } \\
\text { eGFR }\end{array}$ & 12 weeks \\
\hline $\begin{array}{l}\text { Aoike }^{18} \text { 2018, } \\
\text { Brazil }\left(\text { Aoike }^{31}\right. \\
\text { 2015, Baria } \\
\text { 2014, Gomes } \\
\text { 2017) }\end{array}$ & $25 / 15$ & 55.8 & 31.2 & Stage 3-4 & 26.9 & 35 & Usual care & \begin{tabular}{|l|} 
Centre- and \\
home-based \\
aerobic \\
exercise for \\
24 weeks \\
Type: \\
Walking or \\
treadmill \\
Frequency: 3 \\
times/w \\
Intensity: \\
the heart \\
rate value \\
obtained at \\
VT \\
Duration: for \\
30 min with \\
increments \\
of 10 min \\
in duration \\
every 4 weeks \\
until week 8 \\
\end{tabular} & ND & $\begin{array}{l}\mathrm{VO}_{2} \text { peak, } \\
\text { eGFR, Cr, } \\
\text { 6MWT }\end{array}$ & 24 weeks \\
\hline $\begin{array}{l}\text { Barcellos }{ }^{20} 2018, \\
\text { Brazil }\end{array}$ & $58 / 51$ & 65 & 29.9 & Stage $2-4$ & 62.6 & 0 & Usual care & \begin{tabular}{|l|} 
Centre-based \\
aerobic and \\
resistance \\
exercises for \\
16 weeks \\
Type: unclear \\
Frequency: 3 \\
times/week \\
Intensity: \\
unclear \\
Duration: \\
unclear \\
\end{tabular} & 63.7 & eGFR, TUG & 16 weeks \\
\hline Conti & & & & & & & & & & & \\
\hline
\end{tabular}




\begin{tabular}{|c|c|c|c|c|c|c|c|c|c|c|c|}
\hline \multirow[b]{2}{*}{ Trials } & \multirow[b]{2}{*}{$\begin{array}{l}N \text { (analyzed } \\
\text { Exp/Con) }\end{array}$} & \multicolumn{5}{|l|}{ Participants } & \multirow[b]{2}{*}{$\begin{array}{l}\text { Control } \\
\text { group }\end{array}$} & \multirow[b]{2}{*}{$\begin{array}{l}\text { Intervention } \\
\text { group }\end{array}$} & \multirow[b]{2}{*}{\begin{tabular}{|l} 
Compliance of \\
intervention \\
group (\%)
\end{tabular}} & \multirow[b]{2}{*}{$\begin{array}{l}\text { Outcome } \\
\text { measures }\end{array}$} & \multirow[b]{2}{*}{$\begin{array}{l}\text { Follow-up } \\
\text { assessment }\end{array}$} \\
\hline & & Age (years) & BMI & CKD stage & $\begin{array}{l}\text { eGFR }(\mathrm{mL} / \\
\mathrm{min} / 1.73 \\
\left.\mathrm{~m}^{2}\right)\end{array}$ & $\begin{array}{l}\text { Percentage } \\
\text { of DM (\%) }\end{array}$ & & & & & \\
\hline $\begin{array}{l}\text { Beetham }^{19} \\
\text { 2018, Australia } \\
\text { (Howden }^{15,35} \\
\text { 2013, 2015, } \\
\text { Small }{ }^{26} 2017 \text { ) }\end{array}$ & $74 / 68$ & 63.5 & 33.1 & Stage 3-4 & 40.5 & 42.2 & Usual care & \begin{tabular}{|l|} 
Centre-based \\
aerobic and \\
resistance \\
exercises \\
for 8 weeks, \\
followed by \\
home-based \\
aerobic and \\
resistance \\
exercise for \\
10 months \\
Type: \\
Aerobic, \\
treadmill, \\
stationary \\
bike, rowing \\
ergometer \\
Resistance, \\
machine, free \\
weight \\
Frequency: \\
$2-3$ times $/$ \\
week \\
Intensity: \\
moderate \\
inten- \\
sity, with \\
perceived \\
exertion of \\
$11-13$ on \\
the 20-point \\
Borg scale \\
Duration: \\
150 min per \\
week \\
\end{tabular} & ND & $\begin{array}{l}\mathrm{VO}_{2} \text { peak, } \\
\text { eGFR, Cr, } \\
\text { 6MWT, } \\
\text { TUG }\end{array}$ & 52 weeks \\
\hline $\begin{array}{l}\text { Ikizler }^{21} 2018, \\
\text { USA }\end{array}$ & $46 / 46$ & 60 & 33 & Stage 3-4 & 41 & 25 & $\begin{array}{l}\text { Usual physi- } \\
\text { cal activ- } \\
\text { ity + usual } \\
\text { diet }\end{array}$ & \begin{tabular}{|l|} 
Centre-based \\
aerobic \\
exercises for \\
4 months \\
Type: \\
Aerobic; a \\
treadmill, \\
an elliptical \\
cross trainer, \\
a Nu-Step \\
cross trainer, \\
and a \\
recumbent \\
stationary \\
bicycle \\
Frequency: 3 \\
times/week \\
Intensity: \\
$60-80 \%$ VO \\
max \\
Duration: \\
$30-45$ min
\end{tabular} & 85 & $\begin{array}{l}\mathrm{VO}_{2} \text { peak, } \\
\text { eGFR, } \mathrm{Cr}\end{array}$ & 17 weeks \\
\hline ed & & & & & & & & & & & \\
\hline
\end{tabular}




\begin{tabular}{|c|c|c|c|c|c|c|c|c|c|c|c|}
\hline \multirow[b]{2}{*}{ Trials } & \multirow[b]{2}{*}{$\begin{array}{l}\mathrm{N} \text { (analyzed } \\
\text { Exp/Con) }\end{array}$} & \multicolumn{5}{|l|}{ Participants } & \multirow[b]{2}{*}{$\begin{array}{l}\text { Control } \\
\text { group }\end{array}$} & \multirow[b]{2}{*}{$\begin{array}{l}\text { Intervention } \\
\text { group }\end{array}$} & \multirow[b]{2}{*}{$\begin{array}{l}\text { Compliance of } \\
\text { intervention } \\
\text { group }(\%)\end{array}$} & \multirow[b]{2}{*}{$\begin{array}{l}\text { Outcome } \\
\text { measures }\end{array}$} & \multirow[b]{2}{*}{$\begin{array}{l}\text { Follow-up } \\
\text { assessment }\end{array}$} \\
\hline & & Age (years) & BMI & CKD stage & $\begin{array}{l}\text { eGFR }(\mathrm{mL} / \\
\min / 1.73 \\
\left.\mathbf{m}^{2}\right)\end{array}$ & $\begin{array}{l}\text { Percentage } \\
\text { of DM (\%) }\end{array}$ & & & & & \\
\hline $\begin{array}{l}\text { Hiraki }^{23} 2017 \\
\text { Japan }\end{array}$ & $14 / 14$ & 68.7 & 23.7 & Stage 3-4 & 39 & 7.1 & Usual care & $\begin{array}{l}\text { Home-based } \\
\text { aerobic and } \\
\text { resistance } \\
\text { exercises for } \\
1 \text { year } \\
\text { Type: Aero- } \\
\text { bic: brisk } \\
\text { walking } \\
\text { Resistance: } \\
\text { handgrip } \\
\text { strengthen- } \\
\text { ing devise, } \\
\text { squat, calf } \\
\text { raise } \\
\text { Frequency: } 3 \\
\text { times/week } \\
\text { Intensity: } \\
\text { midlevel } \\
\text { intensity, } \\
\text { with per- } \\
\text { ceived exer- } \\
\text { tion on the } \\
\text { Borg scale } \\
\text { Duration: } \\
30 \text { min or } \\
\text { completing } \\
8000-10,000 \\
\text { steps/day } \\
\end{array}$ & 70.4 & $\begin{array}{l}\text { Leg muscle } \\
\text { strength, } \\
\text { eGFR }\end{array}$ & 52 weeks \\
\hline $\begin{array}{l}\text { Leehey }{ }^{16} 2016 \text {, } \\
\text { USA }\end{array}$ & $14 / 18$ & 66 & 36.8 & Stage 2-4 & 40 & 100 & $\begin{array}{l}\text { Only a } \\
\text { nutritional } \\
\text { counseling }\end{array}$ & $\begin{array}{l}\text { Centre-based } \\
\text { aerobic and } \\
\text { resistance } \\
\text { exercises for } \\
12 \text { weeks } \\
\text { followed by } \\
40 \text { weeks of } \\
\text { home-based } \\
\text { aerobic and } \\
\text { resistance } \\
\text { exercises } \\
\text { (total dura- } \\
\text { tion of study } \\
52 \text { weeks) } \\
\text { Type: Aero- } \\
\text { bic, interval } \\
\text { training on } \\
\text { a treadmill; } \\
\text { Resistance, } \\
\text { elastic bands, } \\
\text { handheld } \\
\text { weights } \\
\text { or weight } \\
\text { machine } \\
\text { Frequency: } 3 \\
\text { times/w } \\
\text { Intensity: } \\
\text { Aerobic, } \\
\text { almost mod- } \\
\text { erate (>50\% } \\
\text { of total } \\
\text { time), and } \\
\text { the rest was } \\
\text { light or hard } \\
\text { intensity; } \\
\text { Resistance, } \\
\text { none stated } \\
\text { Duration: } \\
\text { Centre- } \\
\text { based, } \\
60 \text { min of } \\
\text { aerobic and } \\
20-30 \text { min } \\
\text { of resistance } \\
\text { training. } \\
\text { Home-based, } \\
60 \text { min trice } \\
\text { weekly or } \\
30 \text { min } 6 \\
\text { times a week }\end{array}$ & ND & $\begin{array}{l}\mathrm{VO}_{2} \text { peak, } \\
\text { eGFR, Cr, } \\
\text { 6MWT, } \\
\text { TUG, leg } \\
\text { muscle } \\
\text { strength }\end{array}$ & 52 weeks \\
\hline
\end{tabular}




\begin{tabular}{|c|c|c|c|c|c|c|c|c|c|c|c|}
\hline \multirow[b]{2}{*}{ Trials } & \multirow[b]{2}{*}{$\begin{array}{l}\mathrm{N} \text { (analyzed } \\
\text { Exp/Con) }\end{array}$} & \multicolumn{5}{|l|}{ Participants } & \multirow[b]{2}{*}{$\begin{array}{l}\text { Control } \\
\text { group }\end{array}$} & \multirow[b]{2}{*}{$\begin{array}{l}\text { Intervention } \\
\text { group }\end{array}$} & \multirow[b]{2}{*}{$\begin{array}{l}\text { Compliance of } \\
\text { intervention } \\
\text { group }(\%)\end{array}$} & \multirow[b]{2}{*}{$\begin{array}{l}\text { Outcome } \\
\text { measures }\end{array}$} & \multirow[b]{2}{*}{$\begin{array}{l}\text { Follow-up } \\
\text { assessment }\end{array}$} \\
\hline & & Age (years) & BMI & CKD stage & $\begin{array}{l}\text { eGFR }(\mathrm{mL} / \\
\mathrm{min} / 1.73 \\
\left.\mathrm{~m}^{2}\right)\end{array}$ & $\begin{array}{l}\text { Percentage } \\
\text { of DM (\%) }\end{array}$ & & & & & \\
\hline $\begin{array}{l}\text { Tang }^{27} \text { 2016, } \\
\text { China }\end{array}$ & $42 / 42$ & 45.1 & 23.6 & Stage $1-3$ & ND & ND & Usual care & \begin{tabular}{|l} 
Home-based \\
aerobic \\
exercises for \\
12 weeks \\
Type: Aero- \\
bic, walking, \\
cycling, \\
jogging \\
Frequency: 3 \\
times/week \\
Intensity: \\
Moderate \\
inten- \\
sity, with \\
perceived \\
exertion of \\
$12-15$ on \\
the $20-$-point \\
Borg scale \\
Duration: \\
20-30 min
\end{tabular} & ND & $6 \mathrm{MWT}$ & 12 weeks \\
\hline $\begin{array}{l}\text { Greenwood }{ }^{28} \\
2015 \text {, UK }\end{array}$ & $8 / 10$ & 53.5 & 28 & Stage 3-4 & 42.1 & 11.1 & Usual care & $\begin{array}{l}\text { Centre- and } \\
\text { home-based } \\
\text { aerobic and } \\
\text { resistance } \\
\text { exercise for } \\
12 \text { months } \\
\text { Type: Aero- } \\
\text { bic, cycling; } \\
\text { Resistance, } \\
\text { weight } \\
\text { machine } \\
\text { Frequency: } 3 \\
\text { times/week } \\
\text { Intensity: } \\
\text { Aerobic, } \\
80 \% \text { HR } \\
\text { reserve with } \\
\text { maximum } \\
\text { heart rate; } \\
\text { Resistance, } \\
80 \% \text { of } 1 R M \\
\text { Duration: } \\
\text { Aerobic, } \\
\text { two } 20 \text {-min } \\
\text { sessions and } \\
\text { eventually } \\
\text { one } 40 \text {-min } \\
\text { session } \\
\text { Resistance: } \\
3 \text { sets } \times 10 \\
\text { repetitions }\end{array}$ & 79.2 & $\begin{array}{l}\mathrm{VO}_{2} \text { peak, } \\
\text { eGFR, Cr }\end{array}$ & 52 weeks \\
\hline
\end{tabular}




\begin{tabular}{|c|c|c|c|c|c|c|c|c|c|c|c|}
\hline \multirow[b]{2}{*}{ Trials } & \multirow[b]{2}{*}{$\begin{array}{l}\mathrm{N} \text { (analyzed } \\
\text { Exp/Con) }\end{array}$} & \multicolumn{5}{|l|}{ Participants } & \multirow[b]{2}{*}{$\begin{array}{l}\text { Control } \\
\text { group }\end{array}$} & \multirow[b]{2}{*}{$\begin{array}{l}\text { Intervention } \\
\text { group }\end{array}$} & \multirow[b]{2}{*}{$\begin{array}{l}\text { Compliance of } \\
\text { intervention } \\
\text { group (\%) }\end{array}$} & \multirow[b]{2}{*}{$\begin{array}{l}\text { Outcome } \\
\text { measures }\end{array}$} & \multirow[b]{2}{*}{$\begin{array}{l}\text { Follow-up } \\
\text { assessment }\end{array}$} \\
\hline & & Age (years) & BMI & CKD stage & $\begin{array}{l}\text { eGFR }(\mathrm{mL} / \\
\min / 1.73 \\
\left.\mathbf{m}^{2}\right)\end{array}$ & $\begin{array}{l}\text { Percentage } \\
\text { of DM (\%) }\end{array}$ & & & & & \\
\hline $\begin{array}{l}\text { Van } \\
\text { Craenenbroeck }^{30} \\
\text { 2015, Belgium }\end{array}$ & $19 / 21$ & 53.2 & 28.3 & Stage $3-4$ & 38.6 & 7.5 & \begin{tabular}{|l|} 
\\
\\
Standard \\
therapy with- \\
out specific \\
instructions \\
about physi- \\
cal activity
\end{tabular} & $\begin{array}{l}\text { Centre- and } \\
\text { home-based } \\
\text { aerobic } \\
\text { exercise for } \\
12 \text { weeks } \\
\text { Type: Aero- } \\
\text { bic, cycling } \\
\text { Frequency: } \\
\text { In the first } \\
2 \text { weeks of } \\
\text { the study } \\
\text { period, } \\
\text { at least } 3 \\
\text { training } \\
\text { sessions were } \\
\text { supervised } \\
\text { in the hos- } \\
\text { pital by an } \\
\text { experienced } \\
\text { medical } \\
\text { doctor. For } \\
\text { the following } \\
2 \text { weeks, a } \\
\text { supervised } \\
\text { training } \\
\text { session was } \\
\text { organized } \\
\text { once a } \\
\text { week.70 or } \\
\text { more train- } \\
\text { ing days for } \\
12 \text { weeks } \\
\text { Intensity: } \\
90 \% \text { of the } \\
\text { heart rate } \\
\text { achieved at } \\
\text { the anaerobic } \\
\text { threshold } \\
\text { on baseline } \\
\text { testing } \\
\text { Duration: } \\
4 \times 10 \text { min }\end{array}$ & 95.4 & $\begin{array}{l}\mathrm{VO}_{2} \text { peak, } \\
\text { eGFR }\end{array}$ & 12 weeks \\
\hline $\begin{array}{l}\text { Watson } 2015^{29} \text {, } \\
\text { UK }\end{array}$ & $18 / 15$ & $\begin{array}{l}\text { Exp:63/ } \\
\text { Con:66* }\end{array}$ & 32.2 & Stage 3b-4 & $\begin{array}{l}\text { Exp:28.5/ } \\
\text { Con:20.5* }\end{array}$ & $\begin{array}{l}\text { Exp:15/ } \\
\text { Con:27 }\end{array}$ & Usual activity & $\begin{array}{l}\text { Centre-based } \\
\text { resistance } \\
\text { exercise for } \\
8 \text { weeks } \\
\text { Type: resist- } \\
\text { ance machine } \\
\text { Frequency: } 3 \\
\text { times/w } \\
\text { Intensity: } \\
70 \% \text { of } 1 R M \\
\text { Duration: } 3 \\
\text { sets } \times 10-12 \\
\text { repetitions }\end{array}$ & 92 & $\begin{array}{l}\text { Leg muscle } \\
\text { strength }\end{array}$ & 8 weeks \\
\hline $\begin{array}{l}\text { Headley }^{34} 2014 \text {, } \\
\text { USA (Headley } \\
\text { 2017, Miele }{ }^{25} \\
\text { 2017) }\end{array}$ & $25 / 21$ & 57.6 & 35.6 & Stage 3 & 47.6 & 4.3 & Usual care & 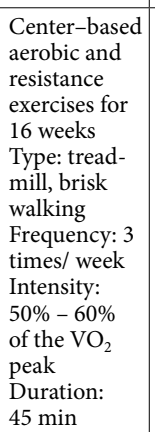 & 96.9 & $\mathrm{VO}_{2}$ peak & 16 weeks \\
\hline
\end{tabular}




\begin{tabular}{|c|c|c|c|c|c|c|c|c|c|c|c|}
\hline \multirow[b]{2}{*}{ Trials } & \multirow[b]{2}{*}{$\begin{array}{l}\mathrm{N} \text { (analyzed } \\
\text { Exp/Con) }\end{array}$} & \multicolumn{5}{|l|}{ Participants } & \multirow[b]{2}{*}{$\begin{array}{l}\text { Control } \\
\text { group }\end{array}$} & \multirow[b]{2}{*}{$\begin{array}{l}\text { Intervention } \\
\text { group }\end{array}$} & \multirow[b]{2}{*}{$\begin{array}{l}\text { Compliance of } \\
\text { intervention } \\
\text { group }(\%)\end{array}$} & \multirow[b]{2}{*}{$\begin{array}{l}\text { Outcome } \\
\text { measures }\end{array}$} & \multirow[b]{2}{*}{$\begin{array}{l}\text { Follow-up } \\
\text { assessment }\end{array}$} \\
\hline & & Age (years) & BMI & CKD stage & $\begin{array}{l}\text { eGFR }(\mathrm{mL} / \\
\min / 1.73 \\
\left.\mathbf{m}^{2}\right)\end{array}$ & $\begin{array}{l}\text { Percentage } \\
\text { of DM (\%) }\end{array}$ & & & & & \\
\hline $\begin{array}{l}\text { Rossi }{ }^{32} 2014, \\
\text { USA }\end{array}$ & $48 / 46$ & 68.5 & 31.5 & Stage 3-4 & ND & 41.1 & $\begin{array}{l}\text { Only stand- } \\
\text { ard CKD } \\
\text { clinic care }\end{array}$ & $\begin{array}{l}\text { Center-based } \\
\text { aerobic and } \\
\text { resistance } \\
\text { exercise for } \\
12 \text { weeks } \\
\text { Type: Aero- } \\
\text { bic; treadmill } \\
\text { walking and/ } \\
\text { or stationary } \\
\text { cycling } \\
\text { Resistance; } \\
\text { free weights } \\
\text { (upper } \\
\text { and lower } \\
\text { extremity) } \\
\text { Frequency: } 2 \\
\text { times/week } \\
\text { Intensity: } \\
\text { Aerobic, a } \\
\text { RPE corre- } \\
\text { sponding to } \\
\text { a } 60 \%-65 \% \\
\text { predicted } \\
\text { maximal } \\
\text { heart rate. } \\
\text { Resistance, } \\
\text { using 1-10- } \\
\text { lb. weights } \\
\text { (according to } \\
\text { tolerance) } \\
\text { Duration: } \\
\text { Aerobic, } \\
60 \text { min; } \\
\text { Resistance, } \\
\text { three sets of } \\
15 \text { repetitions }\end{array}$ & 72.9 & $6 \mathrm{MWT}$ & 12 weeks \\
\hline $\begin{array}{l}\text { Headley }{ }^{36} 2012 \text {, } \\
\text { USA (Gregory }{ }^{37} \\
\text { 2011) }\end{array}$ & $10 / 11$ & 54.9 & 33.5 & Stage $2-4$ & 41.2 & 33.3 & $\begin{array}{l}\text { Standard of } \\
\text { care }\end{array}$ & $\begin{array}{l}\text { Centre-based } \\
\text { aerobic and } \\
\text { resistance } \\
\text { exercises for } \\
48 \text { weeks } \\
\text { Type: } \\
\text { Aerobic: } \\
\text { treadmill, } \\
\text { cycle ergom- } \\
\text { eter, elliptical } \\
\text { machines, } \\
\text { Stairmaster } \\
\text { Resistance: } \\
\text { machine } \\
\text { weight } \\
\text { Frequency: } \\
\text { Aerobic, } 3 \\
\text { times per } \\
\text { week; Resist- } \\
\text { ance, } 2 \text { times } \\
\text { per week } \\
\text { Intensity: } \\
50 \%-60 \% \\
\text { of the VO }{ }_{2} \\
\text { peak } \\
\text { Duration: } \\
\text { Aerobic, } \\
45 \text { min; } \\
\text { Resistance, } \\
\text { two sets of } \\
10-15 \text { repeti- } \\
\text { tions }\end{array}$ & 83.8 & $\begin{array}{l}\mathrm{VO}_{2} \text { peak, } \\
\text { eGFR }\end{array}$ & 48 weeks \\
\hline Contint & & & & & & & & & & & \\
\hline
\end{tabular}




\begin{tabular}{|c|c|c|c|c|c|c|c|c|c|c|c|}
\hline \multirow[b]{2}{*}{ Trials } & \multirow[b]{2}{*}{$\begin{array}{l}\mathrm{N} \text { (analyzed } \\
\text { Exp/Con) }\end{array}$} & \multicolumn{5}{|l|}{ Participants } & \multirow[b]{2}{*}{$\begin{array}{l}\text { Control } \\
\text { group }\end{array}$} & \multirow[b]{2}{*}{$\begin{array}{l}\text { Intervention } \\
\text { group }\end{array}$} & \multirow[b]{2}{*}{$\begin{array}{l}\text { Compliance of } \\
\text { intervention } \\
\text { group (\%) }\end{array}$} & \multirow[b]{2}{*}{$\begin{array}{l}\text { Outcome } \\
\text { measures }\end{array}$} & \multirow[b]{2}{*}{$\begin{array}{l}\text { Follow-up } \\
\text { assessment }\end{array}$} \\
\hline & & Age (years) & BMI & CKD stage & \begin{tabular}{|l} 
eGFR $(\mathrm{mL} /$ \\
$\mathrm{min} / 1.73$ \\
$\left.\mathrm{~m}^{2}\right)$
\end{tabular} & $\begin{array}{l}\text { Percentage } \\
\text { of DM (\%) }\end{array}$ & & & & & \\
\hline $\begin{array}{l}\text { Mustata }{ }^{38} 2011, \\
\text { Canada }\end{array}$ & $10 / 10$ & $\begin{array}{l}\text { Exp:64, } \\
\text { Con:72.5* }\end{array}$ & 28.3 & Stage 3-4 & $\begin{array}{l}\text { Exp: } 27.0, \\
\text { Con:28.0* }\end{array}$ & 55 & Standard care & \begin{tabular}{|l} 
Centre- and \\
home-based \\
aerobic \\
exercises for \\
12 months \\
Type: \\
Treadmill, \\
cycle ergom- \\
eter, elliptical \\
machines, \\
walking \\
Frequency: \\
Centre, 2 \\
times/week; \\
Home, 3 \\
times/week \\
Intensity: \\
$40 \%-60 \%$ \\
of the VO \\
peak \\
Duration: \\
60 min
\end{tabular} & 80 & $\begin{array}{l}\mathrm{VO}_{2} \text { peak, } \\
\text { eGFR }\end{array}$ & 52 weeks \\
\hline $\begin{array}{l}\text { Leehey }^{39} 2009 \text {, } \\
\text { USA }\end{array}$ & $7 / 4$ & 66 & $\mathrm{ND}$ & Stage $2-4$ & 45.1 & 100 & \begin{tabular}{|l|} 
Standard of \\
care medical \\
treatment for \\
diabetes and \\
CKD
\end{tabular} & $\begin{array}{l}\text { Centre-based } \\
\text { aerobic } \\
\text { exercises } \\
\text { for } 6 \text { weeks } \\
\text { followed by } \\
18 \text { weeks of } \\
\text { home-based } \\
\text { aerobic } \\
\text { exercises } \\
\text { (total dura- } \\
\text { tion of study } \\
24 \text { weeks) } \\
\text { Type: } \\
\text { Treadmill or } \\
\text { walking } \\
\text { Frequency: } 3 \\
\text { times/w } \\
\text { Intensity: } \\
\text { Almost mod- } \\
\text { erate ( }>50 \% \\
\text { of total } \\
\text { time), and } \\
\text { the rest was } \\
\text { light or hard } \\
\text { intensity } \\
\text { Duration: } \\
\text { Center; } \\
40 \text { min } \\
\text { Home; } \\
\text { increase their } \\
\text { step count/ } \\
\text { structured } \\
\text { walk by } 10 \% \\
\text { each week }\end{array}$ & ND & $\mathrm{VO}_{2} \max , \mathrm{Cr}$ & 24 weeks \\
\hline $\begin{array}{l}\text { Castaneda }^{41} \\
\text { 2001, USA } \\
\text { (Castaneda }^{40} \\
\text { 2001) }\end{array}$ & $14 / 12$ & $\begin{array}{l}\text { Exp:65/ } \\
\text { Con:64 }\end{array}$ & 28.1 & ND & 26 & 38 & $\begin{array}{l}\text { A low- } \\
\text { protein diet } \\
\text { plus sham } \\
\text { exercises }\end{array}$ & \begin{tabular}{|l} 
A low-pro- \\
tein diet plus \\
Center-based \\
resistance \\
training \\
Type: \\
machine \\
Frequency: 3 \\
times/w \\
Intensity: \\
$80 \%$ \\
Duration: \\
45 min
\end{tabular} & 91 & $\begin{array}{l}\text { Leg muscle } \\
\text { strength, } \\
\text { eGFR, Cr }\end{array}$ & 12 weeks \\
\hline Continued & & & & & & & & & & & \\
\hline
\end{tabular}




\begin{tabular}{|c|c|c|c|c|c|c|c|c|c|c|c|}
\hline \multirow[b]{2}{*}{ Trials } & \multirow[b]{2}{*}{$\begin{array}{l}N \text { (analyzed } \\
\text { Exp/Con) }\end{array}$} & \multicolumn{5}{|l|}{ Participants } & \multirow[b]{2}{*}{$\begin{array}{l}\text { Control } \\
\text { group }\end{array}$} & \multirow[b]{2}{*}{$\begin{array}{l}\text { Intervention } \\
\text { group }\end{array}$} & \multirow[b]{2}{*}{$\begin{array}{l}\text { Compliance of } \\
\text { intervention } \\
\text { group }(\%)\end{array}$} & \multirow[b]{2}{*}{$\begin{array}{l}\text { Outcome } \\
\text { measures }\end{array}$} & \multirow[b]{2}{*}{$\begin{array}{l}\text { Follow-up } \\
\text { assessment }\end{array}$} \\
\hline & & Age (years) & BMI & CKD stage & $\begin{array}{l}\text { eGFR }(\mathrm{mL} / \\
\mathrm{min} / 1.73 \\
\left.\mathrm{~m}^{2}\right)\end{array}$ & $\begin{array}{l}\text { Percentage } \\
\text { of DM (\%) }\end{array}$ & & & & & \\
\hline $\begin{array}{l}\text { Eidemak }^{42} 1997, \\
\text { Denmark }\end{array}$ & $15 / 15$ & $\begin{array}{l}\text { Exp:42/ } \\
\text { Con:44* }\end{array}$ & ND & ND & $\begin{array}{l}\text { Exp:26/ } \\
\text { Con:24* }\end{array}$ & 0 & Usual care & \begin{tabular}{|l|} 
Home-based \\
aerobic \\
training \\
All patients \\
were fol- \\
lowed for a \\
minimum \\
of 1.5 years \\
or until the \\
need for \\
dialysis or \\
kidney trans- \\
plantation \\
Type: bicycle \\
ergometer, \\
and running, \\
swimming \\
and walking \\
Frequency: \\
every day \\
Intensity: \\
$60-75 \%$ VO \\
max \\
Duration: \\
30 min of \\
bicycling \\
daily or an \\
equal amount \\
of other \\
physical \\
activities
\end{tabular} & ND & $\begin{array}{l}\text { eGFR }{ }^{51} \mathrm{Cr}- \\
\text { EDTA), } \mathrm{VO}_{2} \\
\max \end{array}$ & $\begin{array}{l}\text { A minimum } \\
\text { of } 78 \text { weeks } \\
\text { or until need } \\
\text { of dialysis or } \\
\text { kidney trans- } \\
\text { plantation }\end{array}$ \\
\hline
\end{tabular}

Table 1. Characteristics of the included studies. Values of Age, eGFR were expressed as mean. ${ }^{\star}$ median, ND no data, $\mathrm{VO}_{2}$ oxygen uptakes, $e G F R$ estimated glomerular filtration rate, $\mathrm{Cr}$ creatinine, $6 M W T$ six-minute walk test, TUG timed up and go test, $H R R$ heart rate reserve, $R P E$ rating of perceived exertion, $D M$ diabetes mellitus, $R M$ repetition maximum.

\begin{tabular}{|c|c|c|c|c|c|c|}
\hline Trials & $\begin{array}{l}\text { 1. Randomization } \\
\text { process }\end{array}$ & $\begin{array}{l}\text { 2. Deviations from } \\
\text { intended interventions }\end{array}$ & 3. Missing outcome data & $\begin{array}{l}\text { 4. Measurement of } \\
\text { outcome data }\end{array}$ & $\begin{array}{l}\text { 5. Selection of the } \\
\text { reported results }\end{array}$ & Overall \\
\hline Kirkman 2019, USA & Low risk & Some concerns & High risk & Low risk & High risk & High risk \\
\hline Aoike 2018, Brazil & Low risk & Low risk & Low risk & Low risk & Low risk & Low risk \\
\hline Barcellos 2018,Brazil & Low risk & Low risk & Some concerns & Low risk & High risk & High risk \\
\hline Beetham 2018, Australia & Low risk & Low risk & Low risk & Low risk & Low risk & Low risk \\
\hline Ikizler 2018, USA & Low risk & Low risk & Low risk & Low risk & Low risk & Low risk \\
\hline Hiraki 2017, Japan & Low risk & Low risk & Low risk & Low risk & High risk & High risk \\
\hline Leehey 2016, USA & Low risk & Low risk & Some concerns & Low risk & Low risk & Some concerns \\
\hline Tang 2016, China & Low risk & Low risk & Low risk & Low risk & Some concerns & Some concerns \\
\hline Greenwood 2015, UK & Some concerns & Low risk & Low risk & Low risk & High risk & High risk \\
\hline $\begin{array}{l}\text { Van Craenenbroeck 2015, } \\
\text { Belgium }\end{array}$ & Low risk & Low risk & Some concerns & Low risk & Low risk & Some concerns \\
\hline Watson 2015 , UK & Low risk & Low risk & Low risk & Low risk & Some concerns & Some concerns \\
\hline Headley 2014, USA & Low risk & Low risk & Low risk & Low risk & Low risk & Low risk \\
\hline Rossi 2014, USA & Some concerns & Low risk & High risk & Low risk & Low risk & High risk \\
\hline Headley 2012, USA & Some concerns & Low risk & High risk & Low risk & Some concerns & High risk \\
\hline Mustata 2011, Canada & Low risk & Low risk & Low risk & Low risk & Some concerns & Some concerns \\
\hline Leehey 2009, USA & Low risk & Low risk & Some concerns & Low risk & Some concerns & Some concerns \\
\hline Castaneda 2001, USA & Some concerns & Low risk & Low risk & Low risk & High risk & High risk \\
\hline Eidemak 1997, Denmark & Some concerns & Low risk & Low risk & High risk & Some concerns & High risk \\
\hline
\end{tabular}

Table 2. Risk of bias summary.

predicted direction of bias due to missing outcome data. Only 1 trial ${ }^{42}$ showed different time points of measurements in the outcome data because all patients were followed for a minimum of 1.5 years or until the necessity for dialysis or kidney transplantation, which were possibilities of measurement bias. Seven trials ${ }^{16,18,19,21,30,32,36}$ (within all reported outcomes) had enlisted in clinical trial registries or study protocols, whereas 5 trials $s^{8,17,20,28,41}$ 


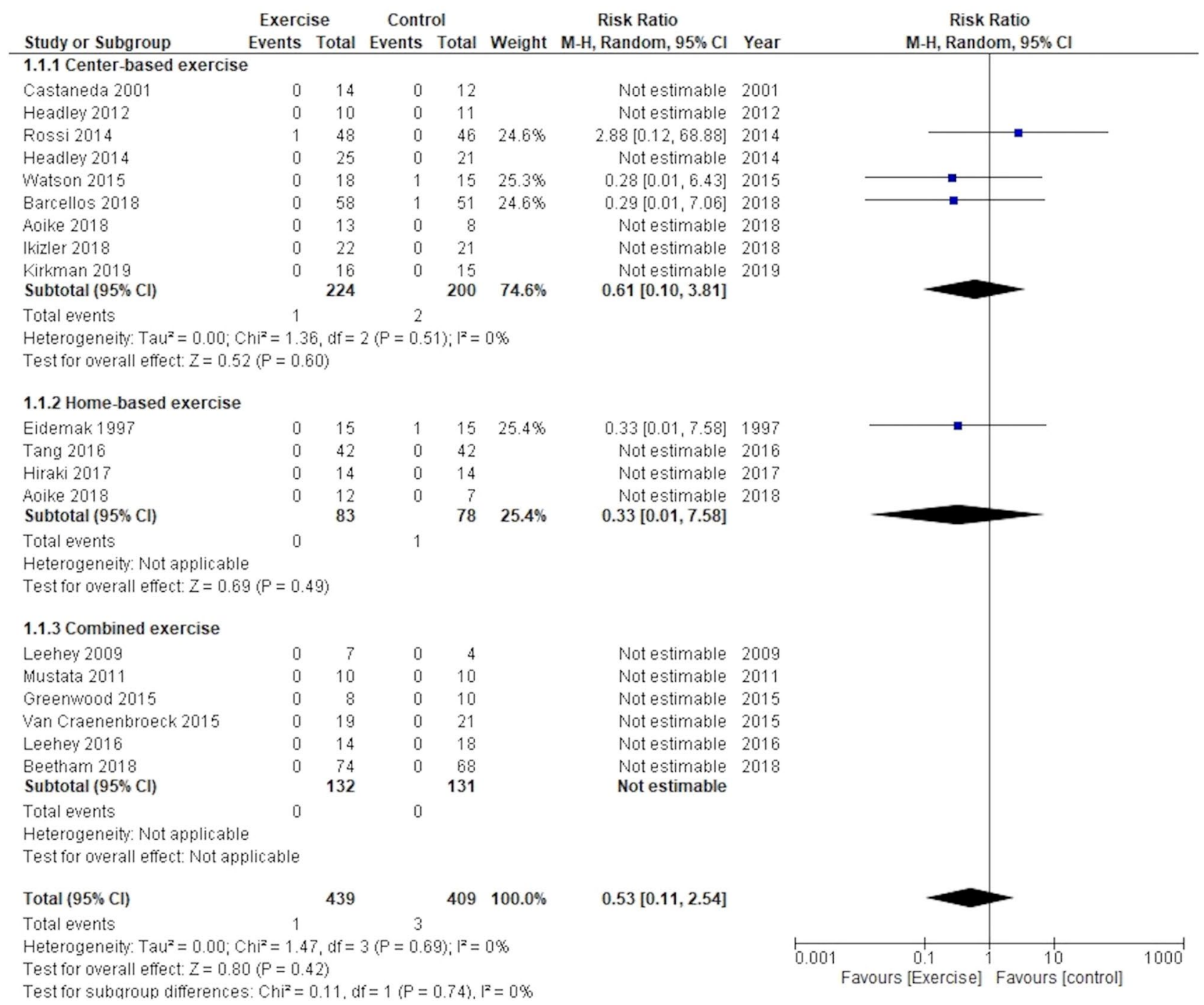

Figure 2. Effect of exercise training on all-cause mortality.

had high reporting bias. The clinical trial registries or study protocols of other trials could not be ascertained; thus, reporting bias was unclear.

Effects of interventions: primary outcomes. All-cause mortality. Eighteen trials, including 848 participants, reported all-cause mortality of the follow-up duration. The effect of physical exercise training on allcause mortality was observed to be uncertain compared to usual care (risk ratio (RR), 0.53 ; $95 \%$ confidence interval $(\mathrm{CI}), 0.11-2.54$; participants $=848$, trials $=18$; $\mathrm{I}^{2}=0 \%$ ) (Fig. 2). Overall, 4 trials ${ }^{20,29,32,42}$ reported the death of participants; however, those were not related to exercise training. Subgroup analysis performed for exercise types, estimated glomerular filtration rate (eGFR) $\left(<30\right.$ or $\left.\geq 30 \mathrm{~mL} / \mathrm{min} / 1.73 \mathrm{~m}^{2}\right)$, and body mass index (BMI) $\left(<30\right.$ or $\geq 30 \mathrm{~kg} / \mathrm{m}^{2}$ ) showed no evidence of differences between the groups (test for subgroup difference: $\mathrm{P}=0.74,0.49$, and 0.68 , respectively). Subgroup analysis was not performed for other variables because an $\mathrm{I}^{2}>50 \%$ was not obtained.

Kidney function (eGFR, Scr). Nine trials including 459 participants reported eGFR as outcomes. eGFRs were mostly evaluated using the CKD-Epidemiology Collaboration (EPI) creatinine equation ${ }^{18,20,28}$ or the Modification of Diet in Renal Disease (MDRD) formula $8,16,17,19,30,36$. The results showed that the effect of exercise training on eGFR was not significant compared to usual care (mean difference (MD), -0.34 ; $95 \% \mathrm{CI}-1.91$ to 1.22 ; participants $=459$, trials $\left.=9 ; \mathrm{I}^{2}=0 \%\right)$ (Fig. 3 ). Subgroup analysis performed for exercise type, eGFR $(<30 \mathrm{or} \geq 30 \mathrm{~mL} /$ $\left.\mathrm{min} / 1.73 \mathrm{~m}^{2}\right)$, and BMI $\left(<30\right.$ or $\left.\geq 30 \mathrm{~kg} / \mathrm{m}^{2}\right)$ showed no evidence of differences between the groups (test for subgroup difference: $\mathrm{P}=0.88,0.19$, and 0.58 , respectively). Subgroup analysis was not performed for other variables because an $\mathrm{I}^{2}>50 \%$ was not obtained.

Only 5 trials including 231 participants reported serum creatinine $(\mathrm{Scr})(\mu \mathrm{mol} / \mathrm{L})$ as outcomes. There was no evidence of effects of physical exercise interventions on Scr improvement compared to usual care (MD, 1.48; $95 \%$ CI -7.50 to 1.31; participants $=231$, trials $=10 ; I^{2}=0 \%$ ) (see Supplementary Fig. S1 online). 


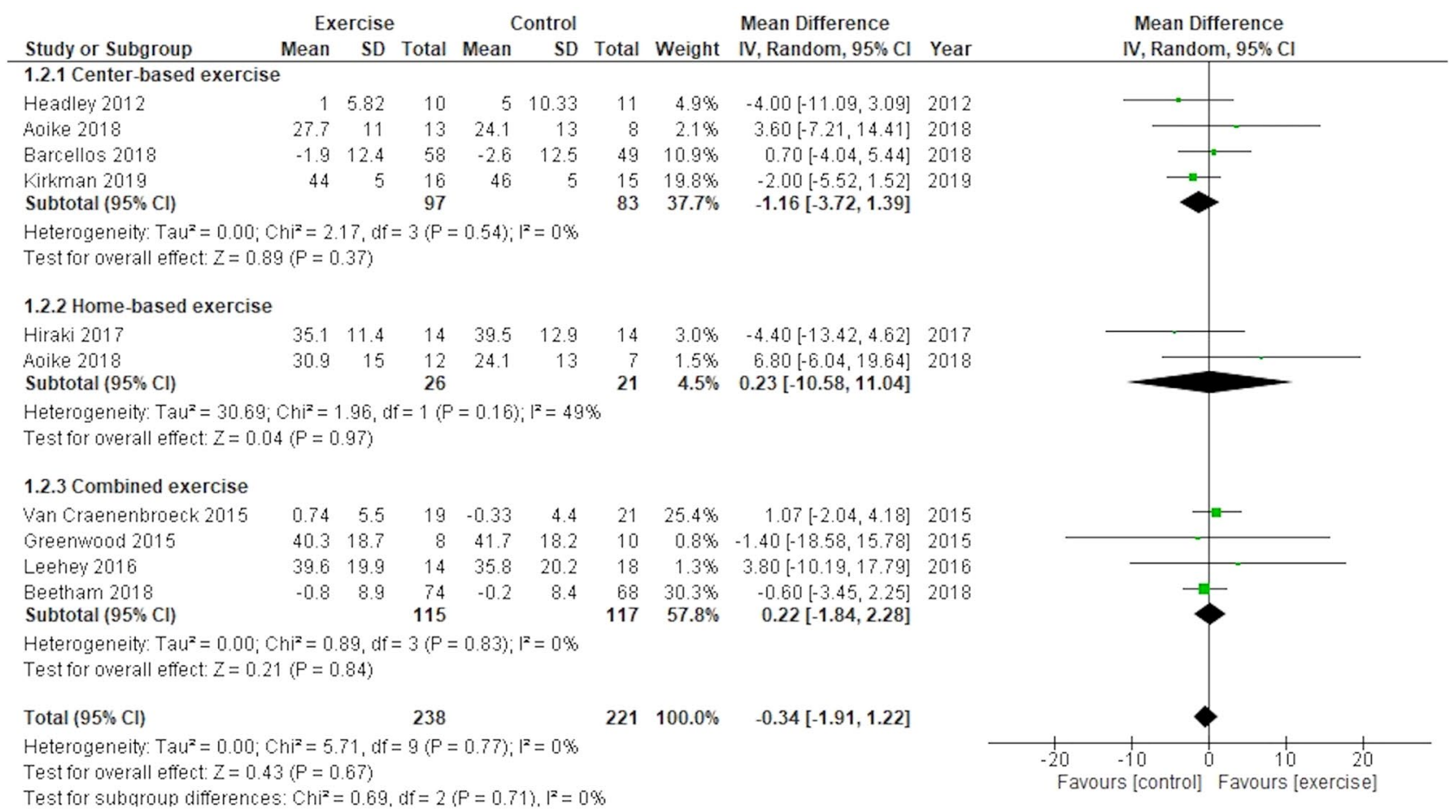

Figure 3. Effect of exercise training on estimated glomerular filtration rate.

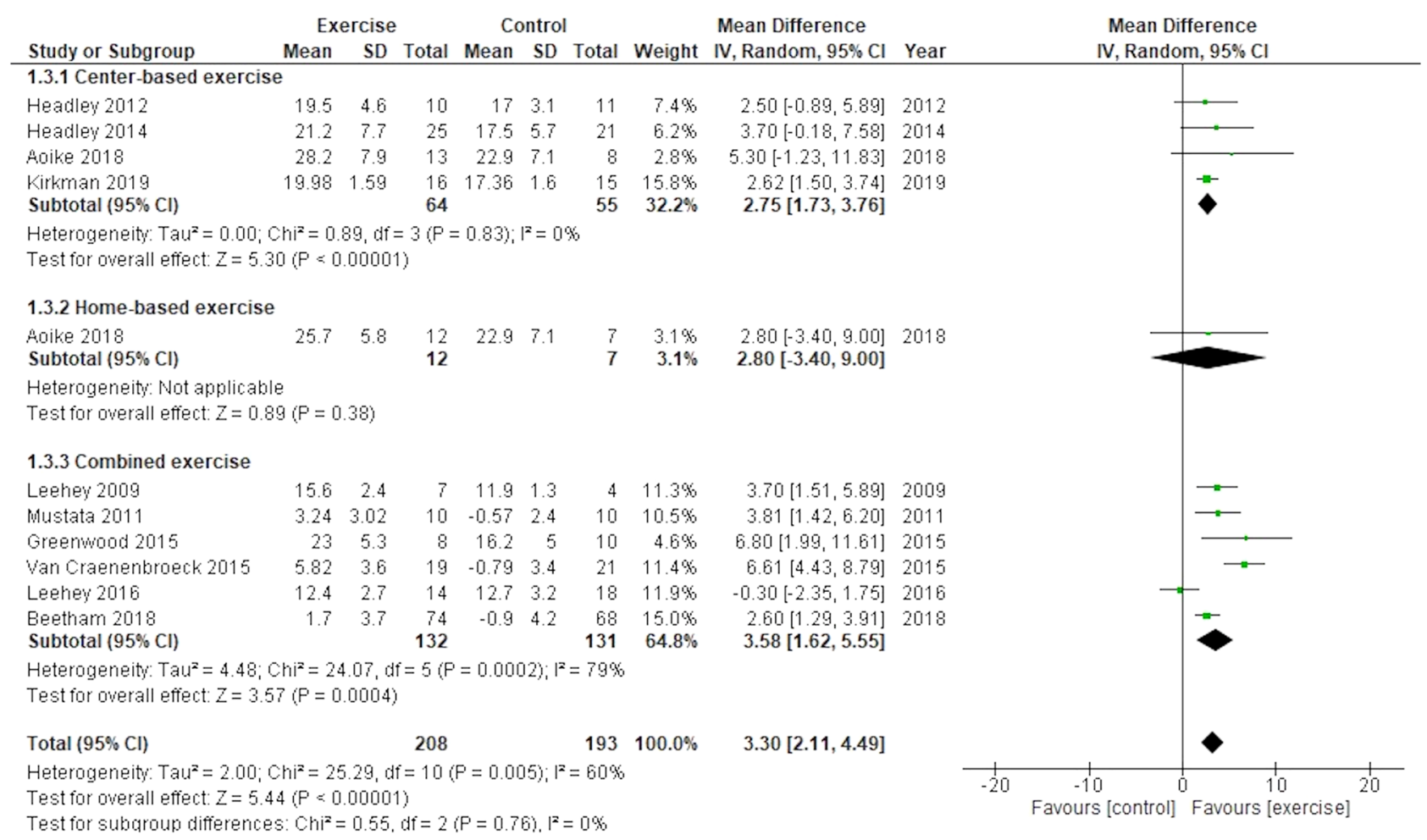

Figure 4. Effect of exercise training on peak/maximum oxygen uptakes.

Physical capacity. Ten trials including 401 participants reported peak/maximum oxygen uptakes (peak/max $\mathrm{VO}_{2}$ ) as physical capacity outcomes. The results demonstrated significant improvements in the peak/max $\mathrm{VO}_{2}$ in the exercise group compared with the usual care group $(\mathrm{MD}, 3.30$; 95\% CI 2.11-4.49; participants $=401$, trials $=10 ; \mathrm{I}^{2}=60 \%$ ) (Fig. 4), although high heterogeneity was detected $\left(\mathrm{P}=0.005, \mathrm{I}^{2}=60 \%\right)$. Subgroup analysis performed for each exercise type, eGFR $\left(<30\right.$ or $\left.\geq 30 \mathrm{~mL} / \mathrm{min} / 1.73 \mathrm{~m}^{2}\right)$, length of exercise intervention, and the 
percentage of patients with diabetes mellitus (DM) complications ( $\geq 50 \%$ or $<50 \%)$ showed no evidence of differences between the groups (test for subgroup difference: $\mathrm{P}=0.66,0.62,0.69$, and 0.37 , respectively). Studies in which the basal average BMI of participants was $<30 \mathrm{~kg} / \mathrm{m}^{2}(\mathrm{MD}, 5.51 ; 95 \%$ CI 3.45-7.57; participants $=78$, trials $=3 ; \mathrm{I}^{2}=37 \%$ ) showed a more significant treatment effect than studies wherein the average BMI of participants was $\geq 30 \mathrm{~kg} / \mathrm{m}^{2}$ (MD, 2.44; 95\% CI 1.45-13.42; participants $=323$, trials $\left.=7 ; \mathrm{I}^{2}=37 \%\right)$; additionally, heterogeneity was lower in subgroup analysis than in pre-subgroup analysis (see Supplementary Table S1 online). In the meta-regression analyses of 9 trials (excluding one $\operatorname{trial}^{39}$ due inadequate BMI data), a significant association was observed between the MD of peak/max $\mathrm{VO}_{2}$ and $\mathrm{BMI}$ [slope:-0.555 BMI (95\% CI - 0.925 to 0.186), $\mathrm{P}=0.009$ ] (see Supplementary Fig. S5 online). The length of exercise intervention and eGFR were not significantly associated with the $\mathrm{MD}$ of peak/max $\mathrm{VO}_{2}(\mathrm{P}=0.301$ and 0.713 , respectively $)$.

Adverse events. Among the included records, 12 trials reported of adverse events related to exercise training; eleven trials showed no adverse event occurred during exercise training and tests. However, only 1 trial $^{21}$ reported 11 adverse events possibly related to the study ( 6 cases of hypotension, 1 case of knee pain, 1 rapid atrial fibrillation case, 1 case of Achilles tendon pain, 1 case of joint pain, and 1 case of chest pain).

Effects of interventions: secondary outcomes. Leg muscle strength. Only 4 trials, including 119 participants, reported leg muscle strength as a physical function outcome. Methods of evaluating leg muscle strength were different among the 4 trials (Hand-held dynamometer ${ }^{8}$, Biodex system 3 isokinetic testing system $^{16}$, Cybex NORM Isokinetic Dynamometer ${ }^{29}$, Keiser resistance training equipment ${ }^{41}$ ). It was observed that the effect of exercise training on leg muscle strength in patients with non-dialysis CKD compared to usual care was uncertain (standard mean difference $(\mathrm{SMD}), 0.35 ; 95 \% \mathrm{CI}-0.03$ to 0.73 ; participants $=119$, trials $=4$; $\mathrm{I}^{2}=7 \%$ ) (see Supplementary Fig. S2 online).

Six-minute walk distance. Five trials, including 392 participants, reported a 6-min walk distance as the walking capacity. Improvements in 6-min walk distance were observed in the exercise group compared to the usual care group (MD, 47.15; 95\% CI 26.87-67.43; participants $=392$, trials $\left.=5 ; I^{2}=64 \%\right)$ (see Supplementary Fig. S3 online), although high heterogeneity was detected.

Time of TUG. Only 3 trials including 170 participants reported timed up and go (TUG) results as dynamic balance evaluation. The results demonstrated that there were improvements with exercise training compared with usual care $\left(\mathrm{MD},-0.72 ; 95 \% \mathrm{CI}-1.21\right.$ to -0.24 ; participants $=170$, trials $=3 ; \mathrm{I}^{2}=0 \%$ ) (see Supplementary Fig. S4 online).

Sensitivity analysis and publication bias. Sensitivity analysis was performed on the exclusion of studies for high risks of bias in the overall results, and there were no changes evident compared to the overall results. Funnel plots showed that trials evaluating eGFR were symmetrically distributed; on the contrary, the distribution of the plot in trials using peak/max $\mathrm{VO}_{2}$ was slightly asymmetrical (see Supplementary Figs. S6, S7 online). In Egger's test, no significant publication bias was observed in trials using eGFR and peak/max $\mathrm{VO}_{2}(\mathrm{P}=0.955$ and 0.261 , respectively).

\section{Discussion}

To the best of our knowledge, this is the first meta-analysis assessing the effects of physical exercise training on the risk of mortality, physical and kidney function, and adverse events exclusively in patients with non-dialysis CKD. The main findings of this review revealed that the effect of exercise training on all-cause mortality and kidney function could not be established in patients with non-dialysis CKD, while exercise training improved physical and walking capacity.

A previously reported systematic review which had conducted meta-analysis showed that exercise training significantly improved eGFR compared with usual care in patients with non-dialysis CKD ${ }^{43}$. However, the review had included non-RCT studies ${ }^{44,45}$, and some study participants were included more than twice in the meta-analysis; this may have resulted in selection bias and the overestimation of the effect of exercise on kidney function. Some studies showed that exercise training improved vascular function, attenuated the increase in sympathetic nervous system activity, and reduced blood pressure in patients with non-dialysis $\mathrm{CKD}^{17,18,28,33,35,38,44}$. This supports the hypothesis that exercise training could delay the decline in kidney function. However, in our review, the effect of exercise training with moderate intensity on the rate of kidney function decline was found to be inconclusive. The duration of exercise intervention in the included trials may have been insufficient to demonstrate improvement in mortality rates and kidney function. As for non-RCT studies, a retrospective longitudinal cohort study ${ }^{46}$ reported that the completion of renal rehabilitation consisting of aerobic and resistance training for a 12-week period was associated with longer event-free survival during the follow-up period (median 34 months). Also, an observational study ${ }^{47}$ showed that substitution of sedentary activity with light activity, but not with exercise training, was associated with a lower hazard of death in the CKD group. Similarly, a previous study showed that muscle mass and physical activity affected SCr rather than cystatin C; thus, use of cystatin may be an adequate alternative to assess renal function ${ }^{48}$. Two trials ${ }^{19,28}$ included in our review measured cystatin; however, a meta-analysis could not be performed due to the limited number of trials. A future study assessing the effect of longer duration exercise training on mortality and kidney function based on cystatin levels is required. An increase in adverse events in the exercise training group compared with the usual care group could not be determined. Of all studies in this review, only one reported adverse event related to exercise 
training ${ }^{21}$. Therefore, a meta-analysis of adverse events could not be conducted; there may have been low rates of adverse events in exercise training overall.

Regular exercise training improved physical and walking capacity in patients with non-dialysis CKD; this was consistent with the results of a previous systematic review of the effect of exercise training in patients with $\mathrm{CKD}$, including dialysis patients, kidney transplant patients, and heart failure patients ${ }^{13,49-51}$. Common symptoms of these chronic diseases (CKD including non-dialysis, dialysis, and kidney transplant patients, DM, and heart failure) were loss of muscle strength, lack of physical activity, and reduced physical capacity. A previous study found that physical capacity (e.g., peak/max $\mathrm{VO}_{2}$ ) was related to the mortality of patients with $\mathrm{CKD}^{52}$, suggesting that exercise training increased physical capacity and benefited patients with CKD. The results showed that lower $\mathrm{BMI}$ at baseline predicted greater improvements in peak/max $\mathrm{VO}_{2}$, while other factors were not significantly associated with the effect of exercise training.

In a previous study that included patients with heart failure, BMI was not associated with an improvement in physical capacity ${ }^{53}$, which is in contrast with our study result. However, explaining the association between BMI and improvement in physical capacity was difficult because only univariate meta-regression analysis was performed in this study due to the small trial sample size. This limits a concurrent consideration of the influence of other factors. Furthermore, adherence to exercise training was reported in only $67 \%$ of trials. Adherence rate for exercise training ranged from $63 \%$ to $96.9 \%$, possibly affecting its influence on peak $/ \mathrm{max} \mathrm{VO}_{2}$. Further studies should assess the relationships between the effect of peak/max $\mathrm{VO}_{2}$ and other factors.

Leg muscle strength is an important marker of physical function that predicts mortality in patients with CKD receiving dialysis ${ }^{54}$. However, the effect of resistance exercise training on leg muscle strength was not significant because of the small number of trials involving patients with non-dialysis CKD. Previous reviews showed that progressive resistance training significantly improved standardized muscular strength in patients with CKD on dialysis $^{49}$. Further research is required to determine whether resistance training improves leg muscle strength in patients with non-dialysis CKD.

The generalizability of this review was limited by age and cause of kidney disease. CKD is more common in people aged 65 or more years ${ }^{1,55}$, and diabetes and high blood pressure have been considered as causes of kidney disease ${ }^{6,56,57}$. However, the approximate mean age of participants in the included trials ranged from 50 to 65 years. The number of older adults may increase in the future; therefore, further studies should assess the effect of exercise training on elderly patients with CKD.

There are some limitations to this review. First, complete data were not obtained because there were missing data in some trials, despite efforts in reaching out to the authors. For this reason, there is a possibility of presence of predicted direction of bias. Secondly, some trials have a high risk of bias, especially those related to the predicted direction of bias, because of missing outcome data and information bias due to the absence of blinding. More high-quality RCTs are needed to clarify the effects of exercise training. Trials included in our review were mostly studies with short durations of intervention and follow-up periods were less than 1 year. Thus, the duration of exercise intervention may have been insufficient to show an improvement in the mortality rates and a significant association with exercise training in the meta-regression analysis. Our systematic review did not include non-RCTs, because RCTs are more likely to provide unbiased information about the differential effects of alternative health interventions (clearly defined exercise training or usual care) than non-RCTs. Therefore, inclusion of non-RCTs of good quality with longer follow-up periods could potentially alter the results. Finally, adherence to exercise training was not reported in $33 \%$ of trials, and this may have biased the effect of exercise on kidney and physical functions.

\section{Conclusion}

Regular aerobic and/or resistance training improves physical and walking capacity for patients with non-dialysis CKD. The effect on mortality, kidney function, and leg muscle strength is inconclusive. Furthermore, few adverse events related to exercise training were reported, suggesting that regular exercise training with moderate intensity for 8 weeks to 1.5 years may be safe for patients with non-dialysis CKD. Future studies and multi-center RCTs with larger sample sizes and cohorts of elderly people are needed to focus on the effect of resistance training in non-dialysis CKD.

\section{Methods}

Protocol and registration. The protocol was registered on UMIN Clinical Trial Registry (UMIN ID000039799). The meta-analysis was performed following the Preferred Reporting Items for Systematic Reviews and Meta-Analysis (PRISMA) statement ${ }^{58,59}$. A systematic review was conducted in agreement with the recommendations stated in the Cochrane Handbook ${ }^{60}$.

Eligibility criteria. Types of study. We included all RCTs, cluster-RCTs, and cross-over trials that investigated the effects of physical exercise interventions on physical function, kidney disease, and mortality of patients with non-dialysis CKD. RCTs without appropriate control groups, including those lacking usual care treatment arms, were excluded. Similarly, quasi-RCTs were excluded because their allocation of participants to treatments is not randomized.

Participants. Adult participants older than 18 years of age who were diagnosed with CKD were excluded. CKD was defined according to the Clinical Practice Guideline for the Evaluation and Management of Chronic Kidney Disease $^{2}$. CKD was defined based on the following criteria: (1) abnormal kidney structure or function and (2) presence of CKD for more than 3 months with impaired health status. Abnormal kidney function was defined as decreased GFR $\left(<60 \mathrm{~mL} / \mathrm{min} / 1.73 \mathrm{~m}^{2}\right)$ or detection of one or more abnormalities for markers of kidney damage, 
such as (1) albuminuria (albumin excretion rate (AER) $>30 \mathrm{mg} / 24 \mathrm{~h}$; albumin creatinine ratio (ACR) $>30 \mathrm{mg} / \mathrm{g}$ [>3 mg/mmol]), (2) urine sediment abnormalities, (3) electrolyte imbalance and other abnormalities due to tubular disorders, (4) histological abnormalities, (5) structural abnormalities revealed by imaging exams, and (6) history of kidney transplantation ${ }^{2}$. Participants who have undergone renal replacement therapies, such as dialysis or kidney transplant, were excluded.

Types of interventions. Hospital-based or home-based exercise interventions were included if supervised by health professionals or self-training. Similarly, different types of exercise, such as resistance training, aerobic exercise, or both, were included. The interventions were compared to control interventions, such as usual care or no-exercise care, consisting of medical care. Studies with exercise interventions clearly defined for frequency (at least once a week), intensity (using percentage of peak workload/oxygen uptakes, anaerobic threshold, or Borg scales), or duration of exercise (more than one month) were included. Abnormal types of exercise were equally included.

Outcome measures. The primary outcomes were as follows: (1) all-cause mortality; (2) kidney function (eGFR, Scr); (3) physical capacity (peak/max $\mathrm{VO}_{2}$ ); and (4) adverse events. On the contrary, the secondary outcomes were as follows: (1) muscle strength (leg muscle strength); (2) walking capacity (6-min walk distance); and (3) balance outcome (time of TUG test).

Search strategy for the identification of relevant studies. On 16 August 2019, the PubMed, CINAHL, Cochrane Library databases, and Embase were electronically searched for eligible RCTs with no restrictions for date/time, language, document type, or publication status. A search strategy was adapted for use in the course of exploring the aforementioned databases (Online Appendix 1).

Screening the studies. Two authors (KN, TS) independently screened all titles and abstracts for all potential studies against the inclusion criteria. Full reports were obtained for all titles that appeared to meet the inclusion criteria and for those wherein any uncertainty was observed. Subsequently, the two authors screened the full-text reports to determine whether these articles met the inclusion criteria. Reasons for the exclusion of ineligible studies were identified. In case of disagreements, a third reviewer (SY) provided comments and made a final decision. The entire screening process was recorded, and the study selection process is described in the PRISMA flow chart (Fig. 1).

Data extraction. The two reviewers conducted the data extraction from eligible articles according to the recommendations stated in the PRISMA statement ${ }^{58,59}$. Disagreements were resolved by the third reviewer. Study characteristics and clinical outcome measures were extracted. The extracted data included general information (authors, year of publication, location), participant characteristics (sample size, inclusion/exclusion criteria, randomization process and allocation, mean age, gender, and percentage of patients with diabetes), interventions (the type of intervention, intensity, duration, and frequency), outcome measures (all-cause mortality, kidney function, including eGFR and Scr, physical function markers, including peak $\mathrm{VO}_{2}$, muscular leg strength, time of TUG, and 6-min walk distance). The corresponding authors of the included publications were contacted for missing data and further information if considered necessary.

Risk of bias. The risk of bias was assessed using the Revised Cochrane risk-of-bias tool for randomized trials (RoB 2) criteria recommended by the Cochrane Handbook of Systematic Reviews of Interventions $s^{60}$. The domains for risk of bias are (1) randomization process, (2) deviations from intended interventions, (3) missing outcome data, (4) measurement of outcome data and (5) selection of the reported results. The risk of bias was categorized as low, some concerns, or high. After the judgment of all domains, the overall risk of bias was assessed by the judgment of all domains as low, some concerns, or high risk of bias. The two reviewers independently conducted the risk assessment, and the third reviewer resolved disagreements.

Data synthesis strategy. Statistical analysis was performed using the Review Manager Software (RevMan V.5.3) to combine and calculate the effect size for each outcome according to the recommendations set by the statistical guidelines described in the Cochrane Handbook of Systematic Reviews of Interventions ${ }^{60}$. Meta-analyses of the data were performed if eligible studies were sufficiently clinical and statistically homogeneous. Clinical heterogeneity was assessed by considering the between-study variability for specific factors such as age or type of exercise interventions. Statistical heterogeneity was tested using the Chi-square test and the $\mathrm{I}^{2}$ statistic. In the event there was substantial heterogeneity between studies $\left(\mathrm{I}^{2}>50 \%\right.$ or $\left.\mathrm{P}<0.1\right)$, the study design and characteristics of the studies were examined. The possible causes of heterogeneity were explored by conducting sub-group, meta-regression, or sensitivity analyses. Random effect models were applied when appropriate. A meta-analysis was conducted if data were appropriate. Dichotomous data (mortality) were described using risk ratios (RR) with a 95\% confidence interval (CI). Continuous outcomes were analyzed using weighted mean differences (WMD) (with 95\% CI) or SMD (95\% CI) if different measurement scales were used.

Subgroup, meta-regression, or sensitivity analyses. Sub-group analysis was performed on all primary outcomes as follows: exercise types (center-based or home-based exercise, or a combination of center- and home-based exercise), basal eGFR $\left(<30 \mathrm{~mL} / \mathrm{min} / 1.73 \mathrm{~m}^{2}\right.$ or $\left.\geq 30 \mathrm{~mL} / \mathrm{min} / 1.73 \mathrm{~m}^{2}\right)$, and basal BMI $\left(<30 \mathrm{~kg} / \mathrm{m}^{2}\right.$ or $\geq 30 \mathrm{~kg} / \mathrm{m}^{2}$ ). For center-based exercises, patients participated in exercise training sessions under the real-time 
supervision of professionals either in a hospital or training center. For home-based exercises, patients performed exercise training at home or in a community setting without real-time supervision of professionals.

Furthermore, sub-group and meta-regression analyses were performed to explore the causes of heterogeneity among primary outcomes if an $\mathrm{I}^{2}>50 \%$ was obtained. Meta-regression analysis was performed using the Stata 14 software (www.stata.com). The length of exercise intervention ( $<24$ weeks, $24-48$ weeks, or $\geq 48$ weeks) and percentage of patients with DM complications $(\geq 50 \%$ or $<50 \%)$ were used as subgroup factors. In the univariate meta-regression model, eGFR, BMI, or length of intervention were used as independent factors.

Sensitivity analysis was performed to explore the sources of heterogeneity, such as the exclusion of studies with a high risk of bias and the evaluation of meaningful changes in the effect size.

Assessment of publication bias. The potential for publication bias was assessed using funnel plots and Egger's test if more than ten studies were available.

\section{Data availability}

We confirm that the data supporting the findings of this review are available within the article and its supplementary materials.

Received: 21 April 2020; Accepted: 14 October 2020

Published online: 23 October 2020

\section{References}

1. GBD Chronic Kidney Disease Collaboration. Global, regional, and national burden of chronic kidney disease, 1990-2017. a systematic analysis for the Global Burden of Disease Study 2017. Lancet. 395(10225), 709-733. https://doi.org/https://doi.org/10.1016/ S0140-6736(20)30045-3 (2020).

2. Levin, A. et al. Kidney disease: Improving global outcomes (KDIGO) CKD work group: KDIGO 2012 clinical practice guideline for the evaluation and management of chronic kidney disease. Kidney Int. Suppl. 3(1), 1-150. https://doi.org/10.1038/kisup.2012.73 (2013).

3. Go, A. S., Chertow, G. M., Fan, D., McCulloch, C. E. \& Hsu, C. Y. Chronic kidney disease and the risks of death, cardiovascular events, and hospitalization. N. Engl. J. Med. 351(13), 1296-1305. https://doi.org/10.1056/NEJMoa041031 (2004).

4. Masson, P. et al. Chronic kidney disease and the risk of stroke: a systematic review and meta-analysis. Nephrol. Dial Transplant. 30(7), 1162-1169. https://doi.org/10.1093/ndt/gfv009 (2015).

5. Chowdhury, R., Peel, N. M., Krosch, M. \& Hubbard, R. E. Frailty and chronic kidney disease: a systematic review. Arch. Gerontol. Geriatr. 68, 135-142. https://doi.org/10.1016/j.archger.2016.10.007 (2017).

6. Masakane, I. et al. Annual dialysis data report 2016, JSDT renal data registry. Ren. Replace. Ther. 4, 45. https://doi.org/10.1186/ s41100-018-0183-6 (2018).

7. Manns, B. et al. The cost of care for people with chronic kidney disease. Can. J. Kidney Health Dis. 6, 1. https://doi.org/10.1177/20543 58119835521 (2019)

8. Hiraki, K. et al. Decreased physical function in pre-dialysis patients with chronic kidney disease. Clin. Exp. Nephrol. 17(2), 225-231. https://doi.org/10.1007/s10157-012-0681-8 (2013).

9. Reese, P. P. et al. Physical performance and frailty in chronic kidney disease. Am. J. Nephrol. 38(4), 307-315. https://doi. org/10.1159/000355568 (2013).

10. Shlipak, M. G. et al. Elevations of inflammatory and procoagulant biomarkers in elderly persons with renal insufficiency. Circulation 107(1), 87-92. https://doi.org/10.1161/01.cir.0000042700.48769.59 (2003).

11. MacKinnon, H. J. et al. The association of physical function and physical activity with all-cause mortality and adverse clinical outcomes in nondialysis chronic kidney disease: a systematic review. Ther. Adv. Chronic Dis. 9(11), 209-226. https://doi. org/10.1177/2040622318785575 (2018).

12. Heiwe, S. \& Jacobson, S. H. Exercise training for adults with chronic kidney disease. Cochrane Database Syst. Rev. 10, 1. https:// doi.org/10.1002/14651858.CD003236.pub2 (2011).

13. Heiwe, S. \& Jacobson, S. H. Exercise training in adults with CKD: a systematic review and meta-analysis. Am. J. Kidney Dis. 64(3), 383-393. https://doi.org/10.1053/j.ajkd.2014.03.020 (2014).

14. Pei, G. et al. Aerobic exercise in adults with chronic kidney disease (CKD): a meta-analysis. Int. Urol. Nephrol. 51(10), 1787-1795. https://doi.org/10.1007/s11255-019-02234-x (2019).

15. Howden, E. J. et al. Exercise training in CKD: efficacy, adherence, and safety. Am. J. Kidney Dis. 65(4), 583-591. https://doi. org/10.1053/j.ajkd.2014.09.017 (2015).

16. Leehey, D. J. et al. Structured exercise in obese diabetic patients with chronic kidney disease: a randomized controlled trial. Am. J. Nephrol. 44(1), 54-62. https://doi.org/10.1159/000447703 (2016).

17. Kirkman, D. L. et al. Effects of aerobic exercise on vascular function in nondialysis chronic kidney disease: a randomized controlled trial. Am. J. Physiol. Renal. Physiol. 316(5), F898-F905. https://doi.org/10.1152/ajprenal.00539.2018 (2019).

18. Aoike, D. T., Baria, F., Kamimura, M. A., Ammirati, A. \& Cuppari, L. Home-based versus center-based aerobic exercise on cardiopulmonary performance, physical function, quality of life and quality of sleep of overweight patients with chronic kidney disease. Clin. Exp. Nephrol. 22(1), 87-98. https://doi.org/10.1007/s10157-017-1429-2 (2018).

19. Beetham, K. S., Howden, E. J., Isbel, N. M. \& Coombes, J. S. Agreement between cystatin-C and creatinine based eGFR estimates after a 12-month exercise intervention in patients with chronic kidney disease. BMC Nephrol. 19(1), 366. https://doi.org/10.1186/ s12882-018-1146-4 (2018).

20. Barcellos, F. C. et al. Exercise in patients with hypertension and chronic kidney disease: a randomized controlled trial. J. Hum. Hypertens. 32(6), 397-407. https://doi.org/10.1038/s41371-018-0055-0 (2018).

21. Ikizler, T. A. et al. Metabolic effects of diet and exercise in patients with moderate to severe CKD: a randomized clinical trial. $J$. Am. Soc. Nephrol. 29(1), 250-259. https://doi.org/10.1681/ASN.2017010020 (2018).

22. Headley, S. et al. Blood pressure response to acute and chronic exercise in chronic kidney disease. Nephrology (Carlton). 22(1), 72-78. https://doi.org/10.1111/nep.12730 (2017).

23. Hiraki, K. et al. Effects of home-based exercise on pre-dialysis chronic kidney disease patients: a randomized pilot and feasibility trial. BMC Nephrol. 18(1), 198. https://doi.org/10.1186/s12882-017-0613-7 (2017).

24. Gomes, T. S. et al. Effect of aerobic exercise on markers of bone metabolism of overweight and obese patients with chronic kidney disease. J. Ren. Nutr. 27(5), 364-371. https://doi.org/10.1053/j.jrn.2017.04.009 (2017). 
25. Miele, E. M. et al. High-density lipoprotein particle pattern and overall lipid responses to a short-term moderate-intensity aerobic exercise training intervention in patients with chronic kidney disease. Clin. Kidney J. 10(4), 524-531. https://doi.org/10.1093/ckj/ sfx006 (2017).

26. Small, D. M. et al. Effects of exercise and lifestyle intervention on oxidative stress in chronic kidney disease. Redox. Rep. 22(3), 127-136. https://doi.org/10.1080/13510002.2016.1276314 (2017).

27. Tang, Q. et al. Effects of individualized exercise program on physical function, psychological dimensions, and health-related quality of life in patients with chronic kidney disease: a randomized controlled trial in China. Int. J. Nurs. Pract. 23(2), 1. https://doi. org/10.1111/ijn.12519 (2017).

28. Greenwood, S. A. et al. Effect of exercise training on estimated GFR, vascular health, and cardiorespiratory fitness in patients with CKD: a pilot randomized controlled trial. Am. J. Kidney Dis. 65(3), 425-434. https://doi.org/10.1053/j.ajkd.2014.07.015 (2015).

29. Watson, E. L. et al. Progressive resistance exercise training in CKD: a feasibility study. Am. J. Kidney Dis. 66(2), 249-257. https:// doi.org/10.1053/j.ajkd.2014.10.019 (2015).

30. Van Craenenbroeck, A. H. et al. Effect of moderate aerobic exercise training on endothelial function and arterial stiffness in CKD stages 3-4: a randomized controlled trial. Am. J. Kidney Dis. 66(2), 285-296. https://doi.org/10.1053/j.ajkd.2015.03.015 (2015).

31. Aoike, D. T. et al. Impact of home-based aerobic exercise on the physical capacity of overweight patients with chronic kidney disease. Int. Urol. Nephrol. 47(2), 359-367. https://doi.org/10.1007/s11255-014-0894-8 (2015).

32. Rossi, A. P., Burris, D. D., Lucas, F. L., Crocker, G. A. \& Wasserman, J. C. Effects of a renal rehabilitation exercise program in patients with CKD: a randomized, controlled trial. Clin. J. Am. Soc. Nephrol. 9(12), 2052-2058. https://doi.org/10.2215/CJN.11791 $113(2014)$

33. Baria, F. et al. Randomized controlled trial to evaluate the impact of aerobic exercise on visceral fat in overweight chronic kidney disease patients. Nephrol. Dial. Transplant. 29(4), 857-864. https://doi.org/10.1093/ndt/gft529 (2014).

34. Headley, S. et al. Short-term aerobic exercise and vascular function in CKD stage 3: a randomized controlled trial. Am. J. Kidney Dis. 64(2), 222-229. https://doi.org/10.1053/j.jjkd.2014.02.022 (2014).

35. Howden, E. J. et al. Effects of exercise and lifestyle intervention on cardiovascular function in CKD. Clin. J. Am. Soc. Nephrol. 8(9), 1494-1501. https://doi.org/10.2215/CJN.10141012 (2013).

36. Headley, S. et al. Exercise training improves HR responses and V ${ }^{*}$ O2peak in predialysis kidney patients. Med. Sci. Sports Exerc. 44(12), 2392-2399. https://doi.org/10.1249/MSS.0b013e318268c70c (2012).

37. Gregory, S. M. et al. Lack of circulating bioactive and immunoreactive IGF-I changes despite improved fitness in chronic kidney disease patients following 48 weeks of physical training. Growth Horm IGF Res. 21(1), 51-56. https://doi.org/10.1016/j. ghir.2010.12.005 (2011).

38. Mustata, S. et al. Effects of exercise training on physical impairment, arterial stiffness and health-related quality of life in patients with chronic kidney disease: a pilot study. Int. Urol. Nephrol. 43(4), 1133-1141. https://doi.org/10.1007/s11255-010-9823-7 (2011).

39. Leehey, D. J. et al. Aerobic exercise in obese diabetic patients with chronic kidney disease: a randomized and controlled pilot study. Cardiovasc. Diabetol. 8(62), 1. https://doi.org/10.1186/1475-2840-8-62 (2009).

40. Castaneda, C. et al. Resistance training to reduce the malnutrition-inflammation complex syndrome of chronic kidney disease. Am. J. Kidney Dis. 43(4), 607-616. https://doi.org/10.1053/j.ajkd.2003.12.025 (2004).

41. Castaneda, C. et al. Resistance training to counteract the catabolism of a low-protein diet in patients with chronic renal insufficiency. A randomized, controlled trial. Ann Intern Med. 135(11), 965-76. https://doi.org/https://doi.org/10.7326/0003-4819-13511-200112040-00008 (2001).

42. Eidemak, I., Haaber, A. B., Feldt-Rasmussen, B., Kanstrup, I. L. \& Strandgaard, S. Exercise training and the progression of chronic renal failure. Nephron. 75(1), 36-40. https://doi.org/10.1159/000189497 (1997).

43. Zhang, L. et al. Exercise therapy improves eGFR, and reduces blood pressure and BMI in non-dialysis CKD patients: evidence from a meta-analysis. BMC Nephrol. 20(1), 398. https://doi.org/10.1186/s12882-019-1586-5 (2019).

44. Kiuchi, M. G. \& Chen, S. The effect of the physical activity on polymorphic premature ventricular complexes in chronic kidney disease. Kidney Res. Clin. Pract. 36(2), 167-174. https://doi.org/https://doi.org/10.23876/j.krcp.2017.36.2.167 (2017).

45. Toyama, K., Sugiyama, S., Oka, H., Sumida, H. \& Ogawa, H. Exercise therapy correlates with improving renal function through modifying lipid metabolism in patients with cardiovascular disease and chronic kidney disease. J. Cardiol. 56(2), 142-146. https ://doi.org/10.1016/j.jjcc.2010.06.007 (2010).

46. Greenwood, S. A. et al. Mortality and morbidity following exercise-based renal rehabilitation in patients with chronic kidney disease: the effect of programme completion and change in exercise capacity. Nephrol. Dial Transplant. 34(4), 618-625. https:// doi.org/10.1093/ndt/gfy351 (2019).

47. Beddhu, S., Wei, G., Marcus, R. L., Chonchol, M. \& Greene, T. Light-intensity physical activities and mortality in the United States general population and CKD subpopulation. Clin. J. Am. Soc. Nephrol. 10(7), 1145-1153. https://doi.org/10.2215/CJN.08410814 (2015).

48. Baxmann, A. C. et al. Influence of muscle mass and physical activity on serum and urinary creatinine and serum cystatin C. Clin. J. Am. Soc. Nephrol. 3(2), 348-354. https://doi.org/10.2215/CJN.02870707 (2008).

49. Matsuzawa, R. et al. Exercise training in elderly people undergoing hemodialysis: a systematic review and meta-analysis. Kidney Int. Rep. 2(6), 1096-1110. https://doi.org/10.1016/j.ekir.2017.06.008 (2017).

50. Chien, C. L., Lee, C. M., Wu, Y. W., Chen, T. A. \& Wu, Y. T. Home-based exercise increases exercise capacity but not quality of life in people with chronic heart failure: a systematic review. Aust. J. Physiother. 54(2), 87-93. https://doi.org/10.1016/s0004 -9514(08)70041-2 (2008).

51. Calella, P. et al. Exercise training in kidney transplant recipients: a systematic review. J. Nephrol. 32(4), 567-579. https://doi. org/10.1007/s40620-019-00583-5 (2019).

52. Sietsema, K. E., Amato, A., Adler, S. G. \& Brass, E. P. Exercise capacity as a predictor of survival among ambulatory patients with end-stage renal disease. Kidney Int. 65(2), 719-724. https://doi.org/10.1111/j.1523-1755.2004.00411.x (2004).

53. Horwich, T. B. et al. Relation among body mass index, exercise training, and outcomes in chronic systolic heart failure. Am. J. Cardiol. 108(12), 1754-1759. https://doi.org/10.1016/j.amjcard.2011.07.051 (2011).

54. Matsuzawa, R. et al. Relationship between lower extremity muscle strength and all-cause mortality in Japanese patients undergoing dialysis. Phys. Ther. 94(7), 947-956. https://doi.org/10.2522/pti.20130270 (2014).

55. Anand, S., Johansen, K. L. \& Kurella Tamura, M. Aging and chronic kidney disease: the impact on physical function and cognition. J. Gerontol. A Biol. Sci. Med. Sci. 69(3), 315-322. https://doi.org/10.1093/gerona/glt109 (2014).

56. Iwai, T. et al. Diabetes mellitus as a cause or comorbidity of chronic kidney disease and its outcomes: the Gonryo study. Clin. Exp. Nephrol. 22(2), 328-336. https://doi.org/10.1007/s10157-017-1451-4 (2018).

57. Inaguma, D. et al. Risk factors for CKD progression in Japanese patients: findings from the Chronic Kidney Disease Japan Cohort (CKD-JAC) study. Clin. Exp. Nephrol. 21(3), 446-456. https://doi.org/10.1007/s10157-016-1309-1 (2017).

58. Liberati, A. et al. The PRISMA statement for reporting systematic reviews and meta-analyses of studies that evaluate health care interventions: explanation and elaboration. J. Clin. Epidemiol. 62, e1-34. https://doi.org/10.1016/j.jclinepi.2009.06.006 (2009).

59. Moher, D. et al. Preferred reporting items for systematic reviews and meta-analyses: the PRISMA statement. PLoS Med. 6(7), e1000097. https://doi.org/10.1371/journal.pmed.1000097 (2009).

60. Higgins, J. P. T. (editors) et al. Cochrane handbook for systematic reviews of interventions. 2nd ed. Chichester, UK, John Wiley \& Sons (2019). 


\section{Acknowledgements}

We would like to thank all of the investigators and contributors of our study.

\section{Author contributions}

K.N. and T.S. designed the study. Y.T. designed the search strategy and performed the search. K.N., T.S., and S.Y. performed abstract screening, full text screening, data extraction, and risk of bias assessment. K.N. and T.S. performed the statistical analysis. S.Y. and S.A. involved in interpretation of the data and helped to write the first draft. H.H. helped to contact authors for addressing the release of missing data results in research report. K.N. drafted the article. All authors revised the manuscript and read and approved the final manuscript.

\section{Competing interests}

The authors declare no competing interests.

\section{Additional information}

Supplementary information is available for this paper at https://doi.org/10.1038/s41598-020-75405-X.

Correspondence and requests for materials should be addressed to K.N.

Reprints and permissions information is available at www.nature.com/reprints.

Publisher's note Springer Nature remains neutral with regard to jurisdictional claims in published maps and institutional affiliations.

(c) (i) Open Access This article is licensed under a Creative Commons Attribution 4.0 International License, which permits use, sharing, adaptation, distribution and reproduction in any medium or format, as long as you give appropriate credit to the original author(s) and the source, provide a link to the Creative Commons licence, and indicate if changes were made. The images or other third party material in this article are included in the article's Creative Commons licence, unless indicated otherwise in a credit line to the material. If material is not included in the article's Creative Commons licence and your intended use is not permitted by statutory regulation or exceeds the permitted use, you will need to obtain permission directly from the copyright holder. To view a copy of this licence, visit http://creativecommons.org/licenses/by/4.0/.

(C) The Author(s) 2020 